\title{
Anpassung an den Klimawandel als Herausforderung für Biosphärenreservate - das Beispiel Flusslandschaft Elbe-Brandenburg
}

\author{
Sven Rannow ${ }^{1}$ Barbara Warner ${ }^{2}$ (D)
}

Eingegangen: 4. Dezember 2015 / Angenommen: 8. August 2016 / Online publiziert: 24. August 2016

(C) Springer-Verlag Berlin Heidelberg 2016

Zusammenfassung Der Umgang mit den Folgen des Klimawandels stellt Biosphärenreservate als Modellregionen für nachhaltige Entwicklung vor neue Herausforderungen. Obwohl Einflüsse auf Ökosysteme oder Landschaften nur begrenzt steuerbar und schwer absehbar sind, besteht der Auftrag, Klimawandelfolgen strategisch aufzugreifen. Zudem sind zahlreiche Akteure mit unterschiedlichen Perspektiven in Entscheidungen einzubeziehen, die vor dem Hintergrund unsicherer Zukunftsprognosen getroffen werden müssen. Der Beitrag zeigt am Beispiel des UNESCOBiosphärenreservates Flusslandschaft Elbe-Brandenburg, welche konkreten Handlungsfelder sich abzeichnen und welche Arbeitsphasen ein planvoller Umgang mit dem Klimawandel haben kann. Es werden mögliche Maßnahmen für die Themen Landschaftswasserhaushalt, Vegetation, Fauna und Landnutzung aufgezeigt. An der Elbe kommt dem Flussgebietsmanagement eine besondere Bedeutung zu. Die Diskussion möglicher strategischer Handlungsfelder erläutert, welche Rollen die Verwaltung von Biosphärenreservaten in einem Prozess der Anpassung an den Klimawandel einnehmen kann. Die Möglichkeiten und der Umfang, in dem sich Biosphärenreservate zu Modellregionen für die Anpassung an den Klimawandel entwickeln, werden sich in der Praxis nach den vorhandenen Kapazitäten und der Expertise richten.

Dr. Sven Rannow

s.rannow@npa-mueritz.mvnet.de

$\triangle$ Dr. Barbara Warner

warner@arl-net.de

1 Nationalparkamt Müritz, Schloßplatz 3, 17237 Hohenzieritz, Deutschland

2 Akademie für Raumforschung und Landesplanung, Hohenzollernstraße 11, 30161 Hannover, Deutschland
Schlüsselwörter Anpassungsstrategien . Großschutzgebiete $\cdot$ Biosphärenreservate . Flusseinzugsgebiet $\cdot$ Elbe $\cdot$ Landnutzung

\section{Adaptation to climate change as a challenge for biosphere reserves - the example of the river landscape Elbe-Brandenburg}

Abstract Dealing with the effects of climate change poses new challenges for biosphere reserves as model regions for sustainable development. Although the impacts on ecosystems or landscapes can only be managed to a limited extent and are difficult to predict, the effects of climate change should be strategically addressed. Furthermore, it is necessary to involve numerous actors with various perspectives in the decisions that must be reached against a background of uncertain prognoses for the future. The paper uses the example of the UNESCO-Biosphere Reserve Elbe-Brandenburg River Landscape to illustrate the concrete fields of action that are emerging and the possible work phases involved in a planned approach to dealing with climate change. Various measures relevant to landscape water balance, vegetation, fauna and land use are demonstrated. On the Elbe, river basin management is particularly important. The discussion of possible strategic fields of action elucidates the roles that can be assumed by the administrations of biosphere reserves in a process of climate adaptation. The options and the extent to which biosphere reserves can develop into model regions for adaptation to climate change depend in practice on the capacities and expertise available.

Keywords Adaptation strategies - Major protected areas · Biosphere reserves $\cdot$ River basin $\cdot$ Elbe $\cdot$ Land use 


\section{Einleitung}

Biosphärenreservate sollen nach den internationalen Leitlinien der UNESCO (1996; 2008) dazu beitragen, Kultur, Tradition und die lokale biologische Vielfalt zu erhalten. Sie sollen eine nachhaltige gesellschaftliche Entwicklung unterstützen und Bildung für nachhaltige Entwicklung und Forschung sowie Umweltbeobachtung ermöglichen und fördern. Eine nach Schutzintensität gegliederte dreifache Zonierung legt Schwerpunkträume für die Umsetzung dieser Kernaufgaben fest. Verwaltungen der Biosphärenreservate bringen mit ihrer lokalen Fachkenntnis und ihren Netzwerken die Voraussetzungen mit, Anpassungskonzepte anzustoßen und als Moderator umzusetzen. Es wird postuliert, dass diese Modellregionen und Lernorte besonders gut geeignet sind, die anspruchsvollen Übersetzungsleistungen, die für die Vermittlung von Anpassungsbedarfen nötig sind, zu erbringen (BfN 2012).

Auch Biosphärenreservate müssen Klimaschutzziele stärker berücksichtigen (German Commission for UNESCO 2011). Ein planvoller Umgang mit Klimafolgen stellt jedoch die bestehenden Schutzgebietsverwaltungen inhaltlich und bezüglich ihrer Kapazitäten vor neue Herausforderungen (BfN 2012). Anpassungs- und Schutzmaßnahmen im Rahmen des Klimawandels sind komplexe gesellschaftliche Prozesse (Karczmarzyk/Pfriem 2011; BBSR 2014; Knieling/Müller 2015). Biosphärenreservate ebenso wie andere Großschutzgebiete sind schon aufgrund ihres Schutzauftrages von klimatischen Änderungen in besonderer Weise betroffen. Strategien der Adaption von Klimafolgen sind mit vielen Akteuren abzustimmen und haben in unterschiedlicher Intensität Interessen des Naturschutzes sowie die Schutzgebietsziele zu berücksichtigen. Ziele und Strategien der Steuerung sind vor dem komplexen Auftrag der Biosphärenreservate immer wieder neu zu bewerten.

Der Umgang mit den Folgen des Klimawandels ist vielschichtig. Aus dem Auftrag der „Dresden Declaration“ (UNESCO 2011) lassen sich Verknüpfungen zu anderen Aufgaben (z. B. Bewältigung des demographischen Wandels, Ressourcenschutz) ableiten. Die Deklaration legt übergeordnete Ziele zu Klimaschutz und Anpassung an den Klimawandel in Biosphärenreservaten fest und richtet sich an die UNESCO selbst, politische Akteure der Mitgliedstaaten und der Umsetzungspraxis. Eine Operationalisierung der Ziele fehlt allerdings, Klimafolgenbewältigung und -anpassung sind hier noch unklar definiert. Anpassungsziele, die Ansätze für konkrete raum- bzw. klimawirksame Maßnahmen aufzeigen, sind von den Bedingungen vor Ort abhängig und oft aufgrund unsicherer Effekte klimatischer Änderungen schwer zu präzisieren und verbindlich zu formulieren. Das komplexe Thema des Klimawandels ist nur bedingt zu konkretisieren, entsprechend schlecht greifbar und vermittelbar (Renn 2014).
Angemessene Strategien sollen natürliche, soziale oder ökonomische Systeme auf Folgen des Klimawandels vorbereiten, um negative Auswirkungen zu mindern oder Vorteile nutzbar zu machen (IPCC 2007; IPCC 2012). Dazu gehören sowohl die kurzfristige, ungeplante bzw. autonome Reaktion von Akteuren auf spürbare Folgen des Klimawandels wie auch die Planung von längerfristig angelegten Anpassungsmaßnahmen oder die Gestaltung von Entscheidungsabläufen als Vorbereitung auf prognostizierte Veränderungen. Der Umgang mit Klimafolgen und die Entwicklung angemessener Steuerungsinstrumente sind bereits in nicht unter Schutz stehenden Räumen schwierig. Ist ein Gebiet dazu noch mit einem Status versehen, der menschliche Nutzung im Sinne einer nachhaltigen Entwicklung steuern soll, wird es umso schwieriger, bei ungleich größerer Akteursvielfalt Folgewirkungen und konkrete Strategien festzulegen bzw. lenkend einzugreifen (Job/Becken/Paeth 2011). Eine große Herausforderung besteht darin, dass Einflüsse auf Ökosysteme oder Landschaften nur eingeschränkt steuerbar sind, potenzielle Auswirkungen aber in Entwicklungskonzepten aufgegriffen werden müssen, auch wenn sie nicht zuverlässig prognostizierbar sind.

Der vorliegende Beitrag geht den Fragen nach, welche möglichen Klimafolgen im „Biosphärenreservat Flusslandschaft Elbe-Brandenburg" entstehen könnten und welche Aufgaben das Biosphärenreservat bei der Anpassung an den Klimawandel übernehmen kann. Darüber hinaus werden Handlungsfelder für den Umgang mit Klimafolgen in Biosphärenreservaten im Allgemeinen diskutiert und mögliche Verfahrensschritte des Anpassungsprozesses vorgestellt.

\section{Das Biosphärenreservat Flusslandschaft Elbe- Brandenburg}

Das UNESCO-Biosphärenreservat Flusslandschaft Elbe erstreckt sich auf rund 400 Kilometer entlang der Elbe. Es umfasst - fünf Bundesländer übergreifend - eine Gesamtfläche von $3428 \mathrm{~km}^{2} .533 \mathrm{~km}^{2}$ (rund $15 \%$ dieser Fläche) liegen im äußersten Nordwesten Brandenburgs und werden als Biosphärenreservat Flusslandschaft Elbe-Brandenburg durch das Landesamt für Umwelt in Brandenburg verwaltet. Das Gebiet liegt im Norddeutschen Tiefland und umfasst die Niederung am Ostufer entlang von rund 70 Kilometern der unteren Mittelelbe (LAGS 1999: 9).

Das Biosphärenreservat ist Teil des Landkreises Prignitz und gehört mit 36 Einwohnern $/ \mathrm{km}^{2}$ zu den am dünnsten besiedelten Regionen in Deutschland (Landesdurchschnitt Brandenburg: 83 Einwohner $/ \mathrm{km}^{2}$ ). ${ }^{1}$ Die Landschaft wird

\footnotetext{
1 Stand 31. Dezember 2014; Regionaldatenbank, Indikatoren des Themenbereichs Bevölkerung: https://www.regionalstatistik.de/genesis/ online (30.05.2016).
} 
durch die Talsandfläche des Elbe-Urstromtals geprägt. Die Grenze bildet die naturräumliche Einheit der Perleberger Heide, die hauptsächlich durch Kiefernwälder auf sandigen Böden dominiert ist. Sie wird durch die Flüsse Karthane, Stepenitz und Löcknitz durchbrochen, die der Elbe zufließen. Aufgrund des geringen Gefälles und der häufigen Überschwemmungen der Elbe und ihrer Nebenflüsse haben sich in der Aue Niedermoore gebildet. Das Abflussgeschehen in die Elbe prägt die Grundwassersituation im gesamten Biosphärenreservat. Großangelegte Meliorationsmaßnahmen haben ein ausgedehntes Netz aus Gräben und Kanälen hinterlassen. Deiche, Schöpfwerke und Polderflächen ermöglichen heute eine großflächige landwirtschaftliche Nutzung der Elbtalaue (LAGS 1999: 16).

Das Biosphärenreservat hat eine große Bedeutung für den Erhalt auentypischer Pflanzengesellschaften. Besonders wichtig sind dabei die Reste der natürlichen Weich- und Hartholzauwälder ebenso wie anthropogen beeinflusstes Auengrünland (insbesondere Stromtalwiesen und Feuchtgrünland). Gleichzeitig ist das Gebiet von herausragender Bedeutung als Brut- und Rastgebiet für verschiedene bedrohte Vogelarten (z. B. Seeadler, Haliaeetus albicilla; Rotmilan, Milvus milvus; Schwarzstorch, Ciconia nigra; Kiebitz, Vanellus vanellus; Ortolan, Emberiza hortulana). Charakterarten, deren natürliches Vorkommen vorwiegend auf eine Pflanzengesellschaft beschränkt ist, wie Elbebiber (Castor fieber albicus), Fischotter (Lutra lutra), kleine Flussmuschel (Unio crassus) und Schlammpeizger (Misgurnus fossilis), sind im Gebiet regelmäßig vertreten. Insbesondere für den Weißstorch (Ciconia ciconia) hat das Gebiet mit einer Bestandsdichte von zwei Brutpaaren pro $10 \mathrm{~km}^{2}$ und dem Internationalen Storchendorf Rühstädt eine länderübergreifende Bedeutung (LAGS 1999).

\section{Mögliche Klimaveränderungen im Biosphärenreservat}

Der Abschnitt des Elbtals im äußersten Nordwesten Brandenburgs wird durch das mecklenburgisch-brandenburgische Übergangsklima mit Jahresdurchschnittstemperaturen von 7,5-8, $6^{\circ} \mathrm{C}$ geprägt. Durch die relative Nähe zur Nordsee ist das Klima noch eher ozeanisch, das Biosphärenreservat zählt zu den niederschlagreichsten Gebieten Brandenburgs (Scholz 1962: 72). Im Zeitraum 1979-1990 lag die durchschnittliche Niederschlagssumme bei $537 \mathrm{~mm} / \mathrm{a}$ (DWD 1995, zitiert nach MLUR 2002). ${ }^{2}$ Im Mittel wurden 32 Sommertage, sieben heiße Tage und 85 Frosttage ge-

\footnotetext{
${ }^{2}$ Für das Gebiet bestehen keine systematischen Auswertungen der Normalperiode 1961-1990. Es kann nur auf die zitierten Veröffentlichungen des Deutschen Wetterdienstes (DWD) zurückgegriffen werden, die sich auf 1979-1990 beziehen.
}

messen. Die Vegetationsperiode dauerte 234 Tage (DWD 1995, zitiert nach MLUR 2002). Für die Region wird ein Anstieg der durchschnittlichen Jahrestemperatur erwartet (Wechsung/Hartje/Kaden et al. 2013; Prüter/Keienburg/ Schreck 2014), die Höhe unterscheidet sich je nach verwendetem Modell. Das „Statistical Analogue Resampling Scheme“ (STARS) prognostiziert für das hydrologische Sommerhalbjahr im Einzugsgebiet der Elbe im Vergleich der Perioden 2004-2013 und 2044-2053 eine Zunahme um $1,2{ }^{\circ} \mathrm{C}$, während der Mittelwert im Winterhalbjahr um $2,0{ }^{\circ} \mathrm{C}$ ansteigt. Projektionen des ,Inter-Sectoral Impact Model Intercomparison Project" (ISI-MIP) gehen von einer mittleren Erwärmung um $1,6{ }^{\circ} \mathrm{C}$ für beide Halbjahre aus (Roers/Wechsung 2015: 113). Gleichzeitig werden Frosttage ab- und Hitzetage mit mehr als $30^{\circ} \mathrm{C}$ zunehmen.

Für die Niederschläge prognostiziert STARS in den Sommermonaten eine deutliche Abnahme (Juni: $-6 \mathrm{~mm}$, Juli: $-9 \mathrm{~mm}$, August: $-6 \mathrm{~mm}$ ) des Medians der jeweiligen Monatssumme, während das Modell für die Wintermonate eine geringe Zunahme ergibt (Roers/Wechsung 2015: 113). Im Gegensatz dazu zeigen sich in den Modellläufen von ISI-MIP keine so eindeutigen saisonalen Änderungen der mittleren Niederschlagssummen. Insbesondere im Sommer wechseln sich Monate mit sinkenden und steigenden Mittelwerten ab (Roers/Wechsung 2015: 113). Durch die Elbe ist die Region mit dem Witterungsgeschehen im gesamten Einzugsgebiet verbunden. Die Häufung von „Jahrhunderthochwässern" in den letzten zwei Jahrzehnten (2002, 2006, 2011, 2013) zeigt, wie bedeutsam auch Veränderungen bei Häufigkeit und Intensität von extremen Witterungssituationen in anderen Regionen Mittel- und Osteuropas für die Elbtalaue sind. Langfristige Veränderungen im Abflussgeschehen wirken sich deutlich auf den Landschaftswasserhaushalt in der Elbtalaue aus. Im Projekt KLIWAS ${ }^{3}$ (BfG 2013) wurden für das gesamte Einzugsgebiet der Elbe Abfluss- und Klimaprojektionen erarbeitet (Prüter/ Keienburg/Schreck 2014). Der Trend zu einer Abnahme des mittleren jährlichen Gesamtabflusses und der monatlichen Abflüsse im Sommer wird durch Modellvergleiche bestätigt (Roers/Wechsung 2015). Der Vergleich zeigt, dass die Projektionen von Veränderungen des Niederschlagsund Abflussregimes mit größeren Unsicherheiten verknüpft sind als die Modellierung der Temperaturveränderung. Deutliche Unsicherheiten bestehen bei der Projektion von extremen Witterungsereignissen: Auch diese haben in der Region lokal starke Auswirkungen. Insbesondere sommerliche Gewitterzellen und Starkregen haben mehrfach zu erheblichen Schäden geführt. So wurde im Jahr 1993 die Stadt Perleberg durch eine Sturzflut der Stepenitz in Mitleidenschaft gezogen, nachdem im Einzugsgebiet innerhalb

\footnotetext{
${ }^{3}$ Forschungsprogramm KLIWAS des BMVI = Auswirkungen des Klimawandels auf Wasserstraßen und Schifffahrt.
} 
von 36 Stunden mehr als $300 \mathrm{~mm}$ Niederschlag gefallen waren.

\section{Mögliche direkte und indirekte Klimafolgen im Biosphärenreservat}

Die Folgen des Klimawandels äußern sich räumlich sehr differenziert. Es wird großer raumplanerischer Handlungsbedarf gesehen (de Vries 2006; Ritter 2007; Birkmann/ Vollmer/Schanze 2013). Im Rahmen der Anpassung an den Klimawandel sind auf den Planungsraum zugeschnittene Konzepte für einen Koordinierungsprozess zwischen den Akteuren nötig (Fleischhauer/Overbeck/Janssen et al. 2013). In vorliegenden Ansätzen zur Anpassung an den Klimawandel in Großschutzgebieten wird daher der Definition klarer Ziele des Anpassungsprozesses große Bedeutung zugeschrieben (Cross/Zavaleta/Bachelet et al. 2012; Wilke/ Rannow 2014).

Aufgrund der langfristigen Veränderungen in den klimatischen Mittelwerten sowie durch die erwartete Verschiebung von Häufigkeit und Umfang von Extremereignissen können verschiedene direkte und indirekte Folgen auf die Naturausstattung und die Landnutzungen erwartet werden. Bisher wurden die möglichen Klimafolgen für das Biosphärenreservat Flusslandschaft Elbe-Brandenburg nicht systematisch untersucht. Die folgende Abschätzung hat daher keinen Anspruch auf Vollständigkeit und stützt sich insbesondere bei Extremereignissen auf historische Vergleichsfälle. Wo möglich, wurden wissenschaftliche Untersuchungen und Klimaprojektionen zugrunde gelegt.

\subsection{Folgen für den Landschaftswasserhaushalt}

Die enge Verknüpfung des Elbepegels mit dem Grundwasserstand im Urstromtal der Elbe verursacht eine weiträumige Auswirkung auf die gesamte Landschaft (Scholz/Stab/ Dziock et al. 2005). Hinzu kommen Faktoren, die nicht von klimatischen Einflüssen abhängen wie die Stauregulierung der Nebenflüsse, Flächennutzung oder Biotopausstattung, die das Wasserregime maßgeblich beeinflussen. Die erwartete Verschiebung zu niederschlagsarmen Sommern mit extremen Niedrigwasserphasen in der Elbe verschärft die sommerlichen Trockenphasen bis weit ins Hinterland. Durch steigende Temperaturen erhöht sich gleichzeitig die Evapotranspiration der Vegetation, sodass die im Sommerhalbjahr negative klimatische Wasserbilanz weiter sinkt. Häufigere Situationen mit Trockenstress im Sommer und Herbst sind daher zu erwarten (Scharnke/Krüger/Urban et al. 2014).

Unmittelbare Folgen der zunehmenden Niedrigwasserphasen sind bereits bei den Kleingewässern zu beobachten. Insbesondere die sogenannten Qualmwasserbereiche hinter der Deichlinie, in der sich das Drängewasser während eines Hochwassers sammelt, sind hiervon betroffen. Hoch angepasste Arten wie Kiemenfußkrebse (Branchiopoda) sind unmittelbar auf diese temporären Gewässer angewiesen. Gleichzeitig haben die überschwemmten Flächen und Kleingewässer im Frühjahr eine erhebliche Bedeutung für die Vermehrung von Insekten, Fischen und Amphibien. Ein Ausbleiben der Frühjahrshochwässer wie in den Jahren 2014 und 2015 wirkt sich unmittelbar auf die Nahrungskette in der Aue und den Reproduktionserfolg anderer Arten wie beispielsweise den Weißstorch aus. Niederschlagsarme Frühjahre und Sommer führen gleichzeitig zu einem Wasser- und Sauerstoffmangel in den tieferen grundwassergespeisten Altarmen und Bracks. Steigende Temperaturen insbesondere bei Hitzephasen erhöhen die Gefahr von Ausstickungen ${ }^{4}$ in diesen Gewässern.

Nebengewässer wie die Karthane zeigen regelmäßige Unterschreitungen von Mindestabflüssen während des Sommers. Durch die Stauregulierung sind strömungsarme Bereiche mit starkem Makrophytenwachstum und zunehmender Nährstofffracht entstanden. Sie haben sowohl eine steigende Sauerstoffzehrung und ein verändertes Temperaturregime als auch die Ablagerung von Feinsedimenten am Gewässergrund durch das vermehrte Algenwachstum zur Folge (MLUL 2015). Dies hat insbesondere für kaltstenotherme sowie rheophile ${ }^{5}$ Organismen und Arten mit Anspruch an sauerstoffreiches Wasser negative Konsequenzen, die sich mit steigenden Durchschnittstemperaturen verstärken werden.

\subsection{Folgen für die Vegetation}

Klimatische Änderungen sind ein wesentlicher Grund für Veränderungen in der Pflanzenvielfalt (vgl. z. B. Mosbrugger/Brasseur/Schaller et al. 2014). Die Vegetation in weiten Bereichen des Biosphärenreservats Flusslandschaft ElbeBrandenburg ist stark von der Dynamik der Aue abhängig (Scholz/Stab/Dziock et al. 2005).

Ludewig/Hanke/Korell et al. (2014) haben mögliche Auswirkungen des Klimawandels auf die Auenwiesen im Bereich der mittleren Elbe untersucht. Sie zeigen, wie Charakterarten auf zunehmende Trockenheit reagieren. In häufig überfluteten Stromtalwiesen könnte es bei geänderter Häufigkeit von Hochwässern und Temperaturerhöhungen im Zuge des Klimawandels zu Veränderungen in der Vegetation kommen (Scholz/Henle/Dziock et al. 2009; Ludewig/Hanke/Korell et al. 2014). Hier sind mögliche

\footnotetext{
${ }^{4}$ Unter Ausstickung wird ein Sauerstoffmangel im Gewässer, in der Regel begleitet von einem Fischsterben, verstanden.

5 Unter kaltstenotherm werden Organismen, die an niedere Temperaturen gebunden sind, verstanden, unter rheophil Organismen, die an strömendes Wasser gebunden sind.
} 
Veränderungen in den Deckungsverhältnissen der Vegetation zu nennen, die hydrologischen Bedingungen und die Nutzung sind jedoch wesentlicher als klimatische Einflüsse (Ludewig/Hanke/Korell et al. 2014). So ist der Erhalt der dynamischen Hydrologie (mit einer geeigneten Landnutzung) die ,wichtigste Bedingung für den Schutz der charakteristischen Auwiesenarten“ (Ludewig/Hanke/Korell et al. 2014: 34). Generelle Aussagen über ein sich änderndes Artenspektrum zu treffen ist jedoch schwierig, da neben dem Temperatur-, Niederschlags- und Grundwasserregime auch beispielsweise der Stickstoffhaushalt des Bodens die Stromtalwiesen prägen.

Eine besondere Herausforderung ist die Projektion von Auswirkungen des Klimawandels auf Wälder, da die einzelnen Waldtypen unterschiedlich auf den steigenden $\mathrm{CO}_{2}-\mathrm{Ge}$ halt in der Luft sowie die Veränderungen der meteorologischen Bedingungen reagieren (Frischbier/Profft/Hagemann 2014). Gerstengarbe und Werner (1997) verweisen auf steigende Waldbrandrisiken für das Land Brandenburg, hiervon sind insbesondere Kiefernwälder auf trockenen Standorten betroffen. Mit einem gezielten Waldumbau zu naturnäheren Laubwaldbeständen könnte dem steigenden Risiko begegnet werden (Jenssen/Hofmann/Pommer 2007). Eine besondere Herausforderung stellt der Schutz und Erhalt des Hartholzauwaldes unter den Bedingungen des Klimawandels dar. Die Hauptbaumart Stieleiche (Quercus robur) zeigt bereits heute starke Ausfälle durch Trockenstress und Insektenkalamitäten. Zusätzlich erschwert die in geschädigten Beständen auf trockneren Standorten einwandernde Spätblühende Traubenkirsche (Prunus serotina) eine natürliche Verjüngung von Eichen.

\subsection{Folgen für die Fauna}

Die Folgen für die Fauna sind bisher nur in ersten Ansätzen ablesbar. Dittrich und Rödel (2014) zeigen exemplarisch die Bedeutung des saisonalen Wasserangebots für den Moorfrosch (Rana arvalis) auf. Für diese Art ebenso wie für die meisten anderen Amphibien sind staunasse Böden, überflutete Wiesen und temporäre Kleingewässer im Frühjahr überlebenswichtig. Die prognostizierten Trends erhöhen die Gefahr der Austrocknung von Gewässern, bevor die Entwicklung der Kaulquappen abgeschlossen ist, mit den entsprechenden negativen Konsequenzen für den Bestand der Art.

Ebenfalls für die in den Nebenflüssen der Elbe vorkommende und vom Aussterben bedrohte kleine Flussmuschel (Unio crassus) ist eine Verschärfung der Bestandsgefährdung durch steigende Wassertemperaturen, verstärktes Makrophytenwachstum und ein verändertes Niederschlagsregime zu befürchten (Haase 2014). Unio crassus ist eine Indikatorart für besondere Flusstypen des Tieflandes und sowohl auf der Deutschen als auch der Brandenburger Roten
Liste als vom Aussterben bedroht eingestuft. Der Bestand im Biosphärenreservat hat überregionale Bedeutung.

Weitere indirekte Wirkungen werden durch Veränderungen der Vegetation ausgelöst. Schuster (2014) weist in diesem Zusammenhang auf eine zu erwartende Bewuchsverdichtung im Feuchtgrünland durch höhere Temperaturen und früheren Vegetationsbeginn hin. Steigende Stickstofffrachten aus der Luft dürften dieses Phänomen noch verstärken. Es wird vermutet, dass Bewuchsverdichtung ebenso wie die Verschiebung der Vegetationsperiode negative Auswirkungen auf den Reproduktionserfolg von Wiesenbrütern wie den Kiebitz haben (Schuster 2014). Auch wirft das wiederkehrende Massenauftreten von klimabeeinflussten Schädlingen, wie beispielsweise dem Eichenprozessionsspinner (Thaumetopoea processionea), die Frage auf, mit welchen indirekten und nicht voraussagbaren Wirkungen des Klimawandels zu rechnen ist. Hier sind längst nicht alle Wirkungen und Wirkungsketten absehbar.

Generell kann die Vielfalt von Arten und Lebensgemeinschaften als maßgeblicher Einflussfaktor für die Ökosystem-Funktionsfähigkeit gesehen werden. Klimawandel ist dann als ein zusätzlicher Stressfaktor für Ökosysteme zu bewerten, der ,konventionelle“ Bedrohungen durchaus verschärfen kann. Freudenberger/Schluck/Ibisch (2012) vertreten die Theorie, dass unzerschnittene und ungestörte Ökosysteme ein relativ höheres Maß an Resilienz und Anpassungsfähigkeit aufweisen und weniger stark durch klimawandelbeeinflusste Änderungen beeinflusst werden können. Ibisch/Luthardt/Kreft et al. (2014) führen beispielsweise an, dass für ein gutes Puffervermögen gegenüber Störungen die stabile Funktionstüchtigkeit von Ökosystemen notwendig ist.

\subsection{Folgen für die Landnutzung}

Feuchtere Winter oder ein trockenes Frühjahr haben Auswirkungen auf die Bewirtschaftung der landwirtschaftlichen Nutzflächen. Eingeschränkte Befahrbarkeit bei zunehmenden Überschwemmungen kann dann beispielsweise eine Nutzungsänderung nach sich ziehen. Genauso negativ wirkt eine vermehrte Sommertrockenheit, wenn wie in den Jahren 2014 und 2015 Frühjahrshochwässer ausbleiben. Bei naturschutzfachlichen Pflegemaßnahmen zeichnet sich bereits jetzt ein Anpassungsbedarf an die Änderungen von Klimabedingungen und Phänologie ab. So sind Pflegemaßnahmen in den Schilfflächen des Rambower Moors mit der bisherigen Mahdtechnik nur bei gefrorenem Boden möglich. Durch das Ausbleiben ausgeprägter Frostperioden in den letzten Jahren müssen hier andere Methoden der Schilfmahd eingesetzt werden.

Solche Folgewirkungen des Klimawandels sind schwer absehbar, hier ist die Erarbeitung von Modellen bzw. Szenarien mit entsprechenden Schwankungsspielräumen notwen- 

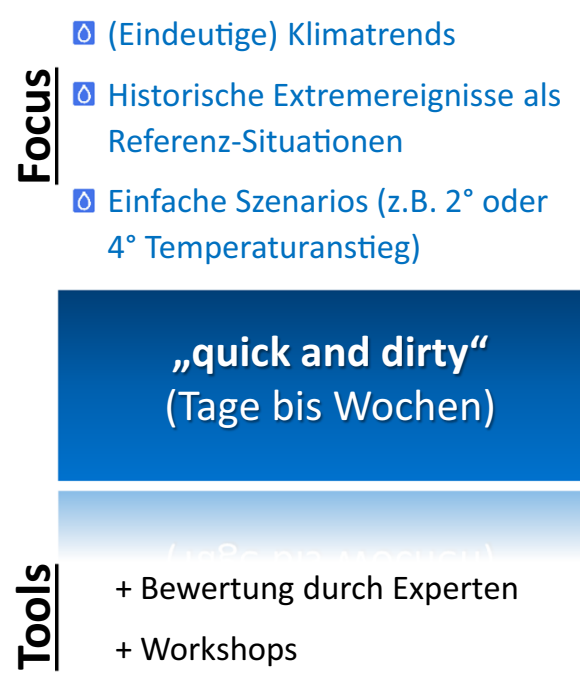
nötigen Arbeitsaufwandes (Quelle: Eigene Darstellung nach Wilke/Rannow 2013: 27)

dig. Da das gesamte Flusseinzugsgebiet bei Anpassungsmaßnahmen zu berücksichtigen ist, wird der Komplexitätsgrad möglicher Strategien erhöht. Hinzu kommen sozioökonomische Auswirkungen auf die Landwirtschaft, die ebenso schon aufgrund der komplexen Wechselwirkungen globaler Verflechtungen schwer prognostizierbar sind.

\section{Umgang mit dem Klimawandel - Mögliche Verfahrensschritte und Bausteine für das Biosphärenreservat Flusslandschaft Elbe- Brandenburg}

Die UNESCO sieht insbesondere in den Bereichen nachhaltige Landnutzung, umweltverträgliches und ressourcenschonendes Wirtschaften, Sicherung von Ökosystemdienstleistungen, Energieeffizienz und Einsatz erneuerbarer Energien wichtige Aufgabenfelder im Umgang mit dem Klimawandel (UNESCO 2011). Eine besondere Herausforderung besteht bei der integrativen Betrachtung der verschiedenen Handlungsfelder. So stellt sich die Frage, ob ökosystembasierte Anpassungskonzepte Win-win-Situationen für den Umgang mit Klimafolgen in mehreren Sektoren liefern könnten (Doswald/Osti 2011).

Bei der Untersuchung von lokalen Veränderungen der klimatischen Rahmenbedingungen und ihrer physischen Folgen können Konzepte wie Risikobetrachtungen oder Vulnerabilitätsanalysen zum Einsatz kommen. Mögliche Methoden unterscheiden sich hinsichtlich ihres Zeitaufwandes sehr. So sind beispielsweise zur Modellierung, die auch auf unterschiedliche räumliche Ebenen Bezug nehmen soll, Monate bis Jahre an Arbeitsaufwand einzurechnen (vgl. Abb. 1).
Neben den in Abb. 1 benannten quantitativen Herangehensweisen sind partizipative Verfahren unabdingbar, auch um bezüglich der Auswirkungen klimatischer Veränderungen den Umgang mit Unsicherheiten gemeinsam zu diskutieren und abgestimmt zu ermöglichen (vgl. Zarzo Fuertes/ Heiland/Rannow et al. 2011; Ibisch/Luthardt/Kreft et al. 2014). Die Involvierung von lokalen Entscheidungsträgern ist als ein zentrales Element der Bewertung und Anpassung $\mathrm{zu}$ sehen, hier greifen ebenso qualitative Analysemethoden. Ibisch/Luthardt/Kreft et al. (2014: 12) nennen hier das Naturschutzmanagement als strategischen Ansatz, möglichst „risiko-robust“ zu agieren.

\subsection{Ziele und Prinzipien von Anpassungsprozessen}

Anpassung an den Klimawandel hat vielfach noch einen Pilot- und Projektcharakter, erfordert Abstraktionsvermögen und Interessensausgleiche bei Unsicherheiten über Folgen klimatischer Veränderungen. Für die Gestaltung von Anpassungsprojekten werden verschiedene Konzepte vorgeschlagen (Hansen/Hoffman 2011; Cross/Zavaleta/Bachelet et al. 2012; European Commission 2012). Der Prozess hängt dabei eng vom betrachteten Handlungsfeld ab, sodass sich nicht jeder Ablauf auf ein beliebiges Anpassungsprojekt übertragen lässt.

Ziel eines Anpassungsprozesses sollte sein, Antworten für das betrachtete Gebiet zu den folgenden Fragen zu erhalten:

- Wo finde ich geeignete und möglichst konkrete Informationen über die potenziellen Auswirkungen des Klimawandels? 
- Welche Konsequenzen ergeben sich hieraus für das jeweilige Handlungsfeld?

- Welche Strategien eignen sich als Reaktion auf die Veränderungen?

Oft wird bei der Analyse ein Schwerpunkt auf die Untersuchung möglichst konkreter Angaben zu lokalen oder regionalen Folgen des Klimawandels gelegt. Potenzielle Verdrängungseffekte und Auswirkungen durch das Auftreten von Klimafolgen an anderer Stelle (z. B. Verlagerung von Tourismusschwerpunkten) werden seltener betrachtet.

\subsection{Arbeitsphasen zur Umsetzung eines Anpassungsprozesses}

Ein strukturierter Anpassungsprozess lässt sich in drei Phasen unterteilen (Wilke/Rannow 2013: 411 ff.). In einer Vorbereitungsphase sollten die Ziele und der Umfang des angestrebten Anpassungsprozesses festgelegt werden. Es sollte geklärt werden, welche Handlungsfelder auf welcher räumlichen Ebene betrachtet werden (einzelne Biotope oder das Schutzgebiet?) sowie die Definition der Ziele und Aufgaben des Prozesses sowie seines Anwendungsbereiches als Voraussetzung für die Methodenwahl. Gleichzeitig sollte die Einbindung relevanter Akteure erörtert werden. Sie ist mit der Herausforderung verbunden, die geeigneten Kommunikationsmittel für die einzelnen Phasen und die zu beteiligenden Akteure zu identifizieren (Zarzo Fuertes/Heiland/ Rannow et al. 2011; Ibisch/Luthardt/Kreft et al. 2014; Wilke/Rannow 2014). Dazu gehört die Projektkommunikation (z. B. Timing, Veranstaltungen), die in dieser Phase konzipiert werden sollte. Vielfach eignet sich für die Vorbereitungsphase ein kleines Team lokaler Experten.

In der Planungsphase sollten sechs Arbeitsschritte vorgesehen werden. Im ersten Schritt wird ein konzeptionelles Schema entworfen, um relevante Einflussfaktoren für das jeweilige Handlungsfeld zu erfassen und Bezüge aufzuzeigen. Hier kann zunächst eine einfache, ausbaufähige graphische Darstellung des Handlungsfeldes genügen. Das Schema dient vor allem zur Wissensdokumentation, zur Diskussion von Einflüssen und Abhängigkeiten sowie zur Identifizierung von wichtigen Stellgrößen. Aufbauend auf diesem Wissen können im nächsten Schritt die nötigen Daten und Informationen gesammelt werden. Hierzu kann die Auswertung historischer Ereignisse ebenso gehören wie die Projektion künftiger Veränderungen. Dieser Arbeitsschritt bindet häufig die meisten Ressourcen, daher muss auf die wichtigen Parameter zur Entscheidungsfindung fokussiert werden. Die benötigten Parameter sind aus dem vormals erstellten Schema abzuleiten (Wilke/Rannow 2014).

Im dritten Schritt sollten die Informationen zu einem transparenten Bild bestehender Trends und möglicher zu- künftiger Klimafolgen zusammengeführt werden. Hier muss abgewogen werden, mit welcher Wahrscheinlichkeit mögliche Folgen eintreffen können. Oft eignen sich hier Szenariotechniken (vgl. Stiens 1977). Dabei müssen die Auswirkungen nicht klimabedingter Entwicklungen miteinbezogen werden (Staudt/Leidner/Howard et al. 2013).

Ein gesonderter Arbeitsschritt sollte die Identifikation von Anpassungsstrategien und Maßnahmen umfassen. Er muss klären, ob eine Strategie zum Schutz und Erhalt, zur Anpassung oder zur aktiven Transformation angestrebt werden sollte (Morecroft/Crick/Duffield et al. 2012). Mit zunehmender Wirkung des Klimawandels kann auch ein Wechsel der Strategien sinnvoll werden. Hierfür müssen die Grenzen für kritische Schwellen einzelner Parameter (z. B. für das Inkrafttreten von Notfallplänen) bestimmt werden.

Weiterhin sollte eine möglichst transparent und abgestimmt verlaufende Prioritätensetzung zur Auswahl von Strategien erfolgen, um spätere Konflikte, die sich aus nicht vorhersehbaren Änderungen ergeben können, angemessen moderieren zu können. Hierbei können die Dringlichkeit, die Realisierbarkeit von Anpassungsmaßnahmen oder die Zuständigkeiten eine Rolle spielen. Die Entwicklung eines Monitoringkonzeptes schließt den Prozess ab. Es soll die Entwicklung des Klimas, den Erfolg von Strategien und Maßnahmen dokumentieren können.

Die Umsetzungsphase umfasst nach Wilke und Rannow (2014: 159 ff.) die Realisierung und gegebenenfalls nötige Anpassung von Maßnahmen. Da sich der Anpassungsprozess über mehrere Jahrzehnte bei sich ändernden Rahmenbedingungen vollziehen wird, ist eine regelmäßige Überprüfung nötig. Neue Klimadaten, verbesserte Klimaprojektionen und neue Erfahrungswerte machen die Anpassung an den Klimawandel zu einem andauernden Prozess (Stein/ Staudt/Cross et al. 2013). Die Grundsätze eines adaptiven, lernenden Managements können daher Anpassungsprozesse unterstützen (Baron/Gunderson/Allen et al. 2009; Heller/ Zavaleta 2009; Ibisch/Luthardt/Kreft et al. 2014).

Ein durch die Verwaltung des Biosphärenreservats gesteuerter Prozess ist elementar. Viele „Ad-hoc“-Lösungen setzen auf technische Maßnahmen, sind langfristig wenig zielführend (Hartmann/Spit 2014) und erhöhen durchaus das Konfliktpotenzial mit Aufgaben von Biosphärenreservaten. Als Beispiel ist hier der technische Hochwasserschutz im Gegensatz zur Schaffung von zusätzlichen Retentionsräumen durch Deichrückverlegungen zu nennen.

\section{Diskussion möglicher Handlungsfelder von Biosphärenreservaten im Rahmen der Anpassung an den Klimawandel}

Die Leistungen der Biosphärenreservate zur Anpassung an die Folgen des Klimawandels sind stärker in Klimastrategi- 
Tab. 1 Handlungsfelder und mögliche Maßnahmen bei Anpassungsstrategien im Biosphärenreservat Flusslandschaft Elbe-Brandenburg

\begin{tabular}{|c|c|}
\hline Handlungsfelder & Mögliche Maßnahmen \\
\hline \multirow[t]{5}{*}{$\begin{array}{l}\text { Landschafts- } \\
\text { wasserhaushalt }\end{array}$} & $\begin{array}{l}\text { Ökosystembasierte Anpassungsmaßnahmen (Wasserrückhalt durch Retentionsräume wie Deichrückverlegungen, Rena- } \\
\text { turierung von Feuchtflächen und Gewässern, gegebenenfalls Einrichtung von Poldern) }\end{array}$ \\
\hline & Förderung angepasster Landnutzung in Poldern \\
\hline & $\begin{array}{l}\text { Dezentraler Hochwasserschutz/Förderung des Wasserrückhaltes in der Fläche zum Schutz bzw. zur Anpassung des } \\
\text { Abflussregimes in Flusseinzugsgebieten }\end{array}$ \\
\hline & Zusammenarbeit mit Planungsverantwortlichen und weiteren Akteuren bei geplanten Nutzungsänderungen \\
\hline & Waldumbau von Nadelholzforsten zu naturnahen Laubwaldbeständen zur Erhöhung der Grundwasserneubildung \\
\hline \multirow[t]{4}{*}{ Vegetation } & Anpassung der Pflegemaßnahmen (z. B. Mahdtermine, Technik) \\
\hline & Schutz von Lebensräumen, Sicherung von Ökosystemleistungen \\
\hline & $\begin{array}{l}\text { Förderung der Resilienz von Ökosystemen (z. B. Erhalt/Verbesserung der Fähigkeit des Waldes zur natürlichen/ } \\
\text { standortgerechten Verjüngung) }\end{array}$ \\
\hline & Wiederherstellung zerstörter Lebensräume (insbesondere Au-, Erlenbruch- und Moorwälder) \\
\hline \multirow[t]{5}{*}{ Fauna } & Ermöglichung der Migration von Arten, gegebenenfalls Erweiterung der Lebensräume \\
\hline & Vernetzung naturnaher Auenlebensräume ${ }^{a}$ \\
\hline & Disaster- und Post-Disaster-Management (z. B. Entschärfung von Problemen durch Biberschutzhügel) \\
\hline & Notfallpläne für nicht ausreichend mobile Arten oder Nutztiere \\
\hline & Priorisierung von Maßnahmen (z. B. Abwägung zwischen Schutzzweck und Allgemeinwohl bei Eingriffen) \\
\hline \multirow[t]{5}{*}{$\begin{array}{l}\text { Landnutzung } \\
\text { (System) }\end{array}$} & $\begin{array}{l}\text { Erhalt von } \mathrm{CO}_{2} \text {-Senken (Erhalt von Wäldern, Mooren und Dauergrünlandflächen in Flusseinzugsgebieten, Anlage von } \\
\text { Weich- und Hartholzauen) }\end{array}$ \\
\hline & Erhalt der Nutzungsfähigkeit von Böden bzw. Bodenfunktionen \\
\hline & Förderung einer umweltverträglichen Bewirtschaftung \\
\hline & Priorisierung von Maßnahmen (Abwägung zwischen Schutzzweck und Allgemeinwohl) \\
\hline & $\begin{array}{l}\text { Verfolgung des Schutzzweckes auch im Oberlauf mit Auswirkungen auf das Biosphärenreservat (z. B. Umgang mit } \\
\text { erhöhten Gewässerbelastungen aufgrund der Intensivierung der Agrarproduktion) }\end{array}$ \\
\hline \multirow{2}{*}{$\begin{array}{l}\text { Nachhaltige } \\
\text { Landnutzung als } \\
\text { Grundlage für das } \\
\text { Gemeinwohl }\end{array}$} & Erhalt und Förderung kultureller Ökosystemleistungen (Erholung, Information, Wissen, Ästhetik etc.) \\
\hline & $\begin{array}{l}\text { Förderung von Naturschutzzielen und -strategien durch Prioritätensetzung/ideelle Stärkung der Naturschutzarbeit, Un- } \\
\text { terstützung von Naturschutzakteuren }\end{array}$ \\
\hline
\end{tabular}

a Zum Auenverbundprojekt an der unteren Mittelelbe vgl. http://www.bund-niedersachsen.de/projekte_einrichtungen/einrichtungen/burg_lenzen/ projekt_auenentwicklung_und_auenverbund/ (23.05.2016)

en $\mathrm{zu}$ integrieren, insbesondere ist die Rolle der Landnutzung bei der Kohlenstoffspeicherung zu fördern (UNESCO 2011: 1 ff.). Es wird davon ausgegangen, dass intakte Ökosysteme gegenüber klimawandelverursachten Änderungen unempfindlicher als bereits beeinträchtigte sind (Naumann/ Davis/Goeller et al. 2015). Dabei hat jedes Ökosystem und jeder Organismus spezifische Ansprüche an das Klima (vgl. Europäische Kommission 2009), außerdem ist landschaftliche Diversität auch in Biosphärenreservaten sehr von der Art und Intensität der Nutzung abhängig. Naumann/Davis/ Goeller et al. (2015: 15 f.) plädieren in diesem Zusammenhang für ökosystembasierte Ansätze bei Maßnahmen, die dem Erhalt und der nachhaltigen Nutzung der Natur dienen, die als ,no-regret options“ auch dann positive Effekte zeigen, wenn vermutete klimabedingte Auswirkungen nicht oder in anderer Form eintreten. Ökosystembasierte Ansätze (z. B. auf ein Flusseinzugsgebiet fokussiert) sind Verwaltungsgrenzen übergreifend angelegt und können existierende Maßnahmen integrieren (vgl. z. B. Hoffmann 2011). Naturschutzfachliche Bewertungskriterien wie „Natürlichkeit" können vor dem Hintergrund von Klimafolgen - die auf einer „unnatürlichen“, da menschenverursachten Grundlage beruhen - gegebenenfalls Neubewertungen erfahren (von Haaren/Galler 2011; von Haaren/Palmas/Siewert et al. 2014; Mosbrugger/Brasseur/Schaller et al. 2014). Beispielgebend ist hier der Umgang mit Neobiota ${ }^{6}$, die aufgrund von Klimaänderungen neue Nischen besetzen und landschaftsprägend werden können.

Mögliche Anpassungsstrategien orientieren sich an Handlungsfeldern, die sich aus dem Schutz- und Entwicklungsauftrag eines Biosphärenreservates ergeben (vgl. Tab. 1). Aus ihnen lassen sich Bedarfe für mögliche Maßnahmen ableiten. So kann aus dem übergeordneten Handlungsfeld Landschaftswasserhaushalt der Bedarf resultieren, Biotope bzw. Flächen für den Überflutungsfall zu sichern. Eine Maßnahme hierzu kann eine Deichrückverlegung sein. Zum anderen resultiert hieraus der Bedarf, neue Retentionsräume zu schaffen. Die möglichen Handlungsfel-

\footnotetext{
${ }^{6}$ Unter Neobiota werden Arten verstanden, die sich über ihre bisherigen Verbreitungsgrenzen ausbreiten und deren Weiterverbreitung in neuen Ökosystemen ermöglicht wird.
} 
Tab. 2 Strategische Handlungsfelder und mögliche Maßnahmen bei Anpassungsstrategien im Biosphärenreservat Flusslandschaft Elbe-Brandenburg

\begin{tabular}{|c|c|}
\hline $\begin{array}{l}\text { Strategische Hand- } \\
\text { lungsfelder }\end{array}$ & Mögliche Maßnahmen \\
\hline $\begin{array}{l}\text { Verwaltung des Bio- } \\
\text { sphärenreservates }\end{array}$ & $\begin{array}{l}\text { Moderation des Diskurses über Anpassungsbedarfe } \\
\text { Konfliktmanagement im Umgang mit Anpassungsmaßnahmen } \\
\text { Anpassung der Konzepte/Richtlinien des Biosphärenreservats } \\
\text { Bereitstellung von Plattformen/Strukturen zur Entwicklung von Anpassungsmaßnahmen } \\
\text { Initiieren und Priorisierung von Anpassungsmaßnahmen }\end{array}$ \\
\hline Umweltbildung & $\begin{array}{l}\text { Modellhaftes Aufzeigen von Klimawirkungen für unterschiedliche Zielgruppen } \\
\text { Kommunikation von Best-Practice-Beispielen für Anpassungsmaßnahmen } \\
\text { Partizipative Umsetzung von Anpassungsmaßnahmen }\end{array}$ \\
\hline $\begin{array}{l}\text { Monitoring/ } \\
\text { Forschung }\end{array}$ & $\begin{array}{l}\text { Forschung zur Änderungen in Artenzahlen und -zusammensetzungen (Welche Faktoren führen zum Artenschwund/ } \\
\text {-erhalt/zur Verschiebung des Artenspektrums?) } \\
\text { Identifikation von No-regret-Optionen, die bei verschiedenen Klimaszenarien wirkungsvoll sind } \\
\text { Autökologischea Untersuchungen zu systemaren Wirkungen des Klimawandels } \\
\text { Forschung zu Auswirkungen des Klimawandels und zur Quantifizierung von Ökosystemleistungen }\end{array}$ \\
\hline
\end{tabular}

${ }^{a}$ Autökologie befasst sich mit den Wechselwirkungen zwischen einer einzigen Art und ihrer Umwelt.

der zur Anpassung an den Klimawandel unterscheiden sich zwischen Großschutzgebieten im lokalen oder kontinentalen Zusammenhang (vgl. Becken/Job 2014). Klimawandel in Biosphärenreservaten bzw. in Großschutzgebieten wird häufig in erster Linie bezüglich der Auswirkungen auf lokale Biodiversität und der nötigen Anpassung des Schutzregimes betrachtet (Schliep/Bertzky/Hirschnitz et al. 2008; Ausden 2014; Macgregor/van Dijk 2014).

Steigende Durchschnittstemperaturen und Veränderungen im Niederschlag erhöhen den Bedarf nach dem Schutz des Landschaftswasserhaushaltes sowie der Schaffung von Feuchtgebieten und Retentionsräumen (Gitay/Finlayson/ Davidson 2011). Insbesondere bei Maßnahmen an oder in Gewässern sollten potenzielle Änderungen in der Wasserverfügbarkeit, im Abflussverhalten oder dem Temperaturregime berücksichtigt werden (Bölscher/van Slobbe/van Vliet et al. 2013). Maßnahmen wirken sich hier wiederum auf andere Sektoren aus und können Einfluss auf den Stellenwert des jeweiligen Schutzgebietes im überregionalen Zusammenhang haben. Die Auswirkungen klimatischer Veränderungen auf Gewässerökosysteme sind in jedem Fall vielschichtiger als auf terrestrische Ökosysteme (Rabitsch/ Winter/Kühn et al. 2010). Ebenso komplex ist es, hier Modelle unter unsicheren Annahmen zu entwickeln. Das trifft auch auf die Region der Elbe zu. Allerdings wären differenzierte Modelle nötig, um Folgen sinkender Grundwasserstände auf die Gewässer abschätzen zu können. Die Modelle sollten Veränderungsszenarien für verschiedene mittlere Grundwasserstände und Jahresganglinien berücksichtigen.

Arten und Lebensräume können sich an starke und rasche klimatische Änderungen nur bedingt anpassen. Lebensgemeinschaften wie beispielsweise bestimmte Waldtypen oder Moore werden sich in ihrer Funktionsfähigkeit verändern. Dies hätte Auswirkungen auch auf Lebensraumfunktionen innerhalb des Schutzgebiets. In diesem Zusammenhang können beispielsweise bezüglich des Handlungsfeldes Vegetation durch Verschiebungen der Jahreszeiten und der Phänologie Anpassungen regelmäßiger Pflegemaßnahmen (Beweidungssysteme, Mahdtermine) nötig werden (Bock/Sparks/Estrella et al. 2013). Beispiele für eine Verlagerung von Lebensräumen und Schaffung neuer Ersatzhabitate lassen sich bereits an den Küsten oder in gezeitengeprägten Regionen finden (Ausden 2014; Ivajnšič/Kaligarič 2014). Die Besiedelung solcher neu geschaffenen Ersatzlebensräume muss allerdings auch möglich sein, das heißt, die Bodenverhältnisse müssen geeignet und die Lebensräume für andere Arten ,zugänglich“ sein sowie Ausbreitungsmöglichkeit bieten.

Gleichzeitig bedroht die Zunahme von Extremwetterlagen (z. B. Stürme, Dürreperioden) die Überlebensfähigkeit von Tier- und Pflanzenbeständen ebenso wie ganzer Ökosysteme. Sie machen die Entwicklung von Notfallplänen und -ketten (z. B. zur Rettung von Weidetieren in halboffenen Weidesystemen bei Hochwasser) nötig. Hierzu gehört an der Elbe die Einrichtung von Biberschutzhügeln im Deichvorland. Auf diese können sich die Tiere bei Hochwasser zurückziehen, ohne den Deich durch Graben von Notbauten zu gefährden. Ebenfalls kann die Vorbereitung einer Katastrophennachsorge (post-disaster management) sinnvoll werden (Haas/Weisz/Pallua et al. 2010).

Offen bleibt, ob durch die Verschiebung von Verbreitungsarealen unter Umständen schutzwürdige Lebensräume neu entstehen. Zusätzlich können Trittsteine oder neue Schutzgebiete nötig werden. Diese dürften neue Anforderungen an die Kohärenz des internationalen Netzwerks und seiner Interaktion mit anderen Großschutzgebieten stellen (Rannow/Macgregor/Albrecht et al. 2014). 
Obwohl damit schon erhebliche Herausforderungen erkennbar werden, geht der Auftrag von Biosphärenreservaten deutlich über den Erhalt von Arten und Biotopen hinaus. Ihre Schutzfunktion bezieht sich auch auf abiotische Elemente, die Kulturlandschaft und Kulturdenkmäler. Die enge Verknüpfung von UNESCO-Biosphärenreservaten und dem Weltkulturerbe macht dies deutlich. Solche Kulturgüter und -monumente werden ebenfalls durch den Klimawandel beeinträchtigt, beispielsweise durch steigende Wasserstände (Marzeion/Levermann 2014). So liegt beispielsweise das Dessau-Wörlitzer Gartenreich im Biosphärenreservat Flusslandschaft Elbe und ist in seinem Status als UNESCO-Weltkulturerbe von den Flusssystemen der Mulde und Elbe und der Auenvegetation geprägt.

Naturschutz konkurriert als Landnutzungssektor stark mit vornehmlich wirtschaftlich motivierten Nutzungen und scheitert somit schnell bei Interessenkonflikten. Daher ist im Sinne einer nachhaltigen Landnutzung auch seine Rolle als Bewahrer des gemeinsamen Erbes zu honorieren und vor allem zu stärken.

\section{Strategische Handlungsfelder}

Über die thematischen Handlungsfelder hinaus ergeben sich auch strategische Aufgaben, um Biosphärenreservate zu Modellregionen für die Klimaanpassung zu entwickeln (vgl. Tab. 2).

Die Verwaltungen von Biosphärenreservaten haben einen konkreten Schutz- und Entwicklungsauftrag, gleichzeitig sind Biosphärenreservate durch eine Vielzahl weiterer Akteure und deren Interessen gekennzeichnet, die teilweise nur indirekt Bezug zu konkreten umweltpolitischen Zielen haben. Das Konstrukt des Biosphärenreservates bedeutet eine Verpflichtung für alle Akteure, sich auf korrespondierende und möglichst nachhaltig ausgestaltete Schutz- und Nutzungskonzepte einzulassen. Dass dies nicht konfliktfrei umsetzbar ist, liegt auf der Hand.

Biosphärenreservatsverwaltungen können je nach Akteurskonstellationen unterschiedliche Rollen bei der Umsetzung der thematischen Handlungsfelder einnehmen. Sie können eigene Maßnahmen umsetzen, geeignete Strukturen schaffen oder Anstöße geben. Ein strategisches Handlungsfeld ist die Vermittlung inhaltlicher Ziele. Hierzu gehört auch die Moderation von Konflikten. Die Herausforderung liegt darin, Nachhaltigkeit in einem Raum mit vorrangig wirtschaftlich agierenden Akteuren zu erproben (UNESCO 2011). Wenn dazu in einem potenziellen „Konfliktraum“ wie einem Biosphärenreservat über unsichere $\mathrm{Zu}$ künfte (Klimafolgen) debattiert werden muss, ist es schwieriger, Maßnahmen mit einer gegebenenfalls eingeschränkten Lobby umzusetzen (z. B. für Naturschutzbelange). Aber auch ohne vollständige Kenntnis der komplexen Wirkungs- zusammenhänge können nachhaltige und kluge Entscheidungen getroffen werden (Schreck/Katz 2014).

Biosphärenreservate sind ,Lernorte für nachhaltige Entwicklung“ (UNESCO 2011: 3, Punkt 12) auch vor dem Hintergrund des großen Problems Klimawandel. Angebote für Kinder und Jugendliche erreichen zwar die ,Umweltbildner von morgen“, es eröffnet sich jedoch ein weites Handlungsfeld auch für die Erwachsenenbildung, die ebenso systemisches Denken und „mündiges Handeln“ initiieren soll (Paulini/Haum 2015). Der Staat hat dabei - im Rahmen der gesellschaftlichen Transformation - Anreize und Raum für Engagement und Experimente zu schaffen, indem er seinen Auftrag als „Gestalter“ wahrnimmt. Bildung sollte die Grundlagen für ein wissenschaftliches Selbstverständnis fördern, das das Individuum befähigt, nachhaltige Entwicklung (mit) zu gestalten (Paulini/Haum 2015). Modellhaftes Aufzeigen von Klimafolgenwirkungen für unterschiedliche Zielgruppen wäre ein erster wichtiger Schritt.

Die Aufgabe, Monitoring und Umweltbeobachtung insbesondere in Kernzonen als Referenzflächen zu betreiben, ist durchaus erweiterbar. Insofern können Biosphärenreservate zur Klimafolgenforschung beitragen. Voraussetzung ist eine systematische Vorgehensweise, die Veränderungen beispielsweise bei Flora und Fauna auf Auswirkungen des Klimawandels zurückführen kann.

Die oben genannten Handlungsfelder als Akteur oder Moderator zu bearbeiten, ist eine umfangreiche Herausforderung für die Verwaltung von Biosphärenreservaten. Sie können dieser Aufgabe nur nachkommen, wenn ausreichende Kapazitäten und die passende Expertise vorhanden sind (BfN 2012). Dies ist derzeit häufig nicht der Fall. Nicht jeder der in Tab. 1 und 2 aufgeführten Handlungsbereiche wird die gleiche Relevanz für ein spezifisches Biosphärenreservat haben. Vielfach wird sich durch die naturräumliche Situation und formale Zuständigkeiten die Notwendigkeit zur Konzentration auf bestimmte Handlungsbereiche ergeben.

\section{Fazit}

Ansätze nachhaltiger Landnutzung und umwelt- und ressourcenschonenden Wirtschaftens sowie die Sicherung von Ökosystemdienstleistungen sollen in Biosphärenreservaten modellhaft entwickelt werden. Hierzu gehört auch die Forschung zu und Evaluierung von Klimaveränderungen und ihrer Auswirkungen sowie die Unterstützung innovativer Instrumente und Strategien zu Klimaschutz und Anpassung an den Klimawandel (UNESCO 2011). Dem Hochwasserschutz kommt dabei in der Elbtalaue eine besondere Bedeutung zu.

Klare Anpassungsziele sind zu definieren, die die Vielzahl der Herausforderungen kanalisieren können und trotz- 
dem die Komplexität des Aufgabenspektrums der Biosphärenreservate widerspiegeln. Für einen Prozess zur Anpassung an den Klimawandel muss analysiert werden, welche Auswirkungen der Klimawandel auf die verschiedenen Aufgaben eines Biosphärenreservats hat und wie mit den Folgen umgegangen werden kann. In den verschiedenen Handlungsfeldern können sich Konsequenzen für den eigenen Aktivitätsbereich, aber auch für die Beeinflussung autonomer Anpassungsstrategien von Landnutzern beispielsweise aus der Land- und Forstwirtschaft ergeben. Gleichzeitig muss betrachtet werden, welche Bedeutung Anpassungsprozesse für die jeweilige Situation eines Biosphärenreservats haben und wo sich entsprechende Maßnahmen in die aktuellen Handlungsprioritäten einfügen. Verfügbare Kapazitäten und die vorhandene Expertise spielen dabei in der Praxis eine erhebliche Rolle. Berücksichtigt werden muss die Akteursvielfalt in Biosphärenreservaten mit ihren Perspektiven und Expertisen. Der Diskurs auf unterschiedlichen Akteursebenen rückt die Komplexität der Entscheidungsfindung in den Fokus, die einerseits die Qualität der Arbeit in Biosphärenreservaten ausmacht, andererseits Entscheidungsprozesse behindert. Hier stehen Biosphärenreservate gerade bei eher unsicheren Datenlagen vor großen Herausforderungen, Schutz, Nutzung und Entwicklung von Natur und Landschaft im Einklang zu fördern.

\section{Literatur}

Ausden, M. (2014): Climate change adaptation: putting principles into practice. In: Environmental Management 54, 4, 685-698.

Baron, J.; Gunderson, L.; Allen, C. D.; Fleishman, E.; McKenzie, D.; Meyerson, L. A.; Oropeza, J.; Stephenson, N. (2009): Options for National Parks and Reserves for Adapting to Climate Change. In: Environmental Management 44, 6, 1033-1042.

BBSR - Bundesinstitut für Bau-, Stadt- und Raumforschung (Hrsg.) (2014): Klimawandel in Stadt und Region. Ergebnisse aus den Forschungsfeldern ImmoKlima/ImmoRisk, StadtKlima und KlimaMORO. Bonn. http://www.bbsr.bund.de/BBSR/DE/Veroeffentlichungen/ Sonderveroeffentlichungen/2014/DL_klimawandel_stadt_region. pdf;jsessionid=4861BFDEC85DD8B3E4C76BEA615CC171. live2052? _blob=publicationFile \& $\mathrm{v}=1(01.07 .2015)$.

Becken, S.; Job, H. (2014): Protected Areas in an Era of Global-Local Change. In: Journal of Sustainable Tourism 22, 4, 507-527.

BfG - Bundesanstalt für Gewässerkunde (2013): Auswirkungen des Klimawandels auf Wasserstraßen und Schifffahrt - Entwicklung von Anpassungsoptionen. Koblenz.

BfN - Bundesamt für Naturschutz (2012): Biosphärenreservate als Modellregionen für Klimaschutz und Klimaanpassung. BonnBad Godesberg. = BfN-Skripte 312.

Birkmann, J.; Vollmer, M.; Schanze, J. (Hrsg.) (2013): Raumentwicklung im Klimawandel. Herausforderungen für die räumliche Planung. Hannover. $=$ Forschungsberichte der ARL 2.

Bock, A.; Sparks, T. H.; Estrella, N.; Menzel, A. (2013): Changes in the timing of hay cutting in Germany do not keep pace with climate warming. In: Global Change Biology 19, 10, 3123-3132.

Bölscher, T.; van Slobbe, E.; van Vliet, M.; Werners, S. (2013): Adaptation Turning Points in River Restoration? The Rhine Salmon Case. In: Sustainability 5, 6, 2288-2304.
Cross, M.; Zavaleta, E.; Bachelet, D.; Brooks, M.; Enquist, C.; Fleishman, E.; Graumlich, L.; Groves, C.; Hannah, L.; Hansen, L.; Hayward, G.; Koopman, M.; Lawler, J.; Malcom, J.; Nordgren, J.; Petersen, B.; Rowland, E.; Scott, D.; Shafer, S.; Shaw, M.; Tabor, G. (2012): The Adaptation for Conservation Targets (ACT) Framework: A tool for incorporating climate change into natural resource management. In: Environmental Management 50, 3, 341-351.

de Vries, J. (2006): Climate Change and Spatial Planning below SeaLevel: Water, Water and more Water, Interface Section. In: Planning Theory and Practice 7, 2, 223-227.

Dittrich, C.; Rödel, M.-O. (2014): Moorfrosch Rana arvalis. In: Kerth, G.; Blüthgen, N.; Dittrich, C.; Dworschak, K.; Fischer, K.; Fleischer, T.; Heidinger, I.; Limberg, J.; Obermaier, E.; Rödel, M.O.; Nehring, S. (Hrsg.): Anpassungskapazität naturschutzfachlich wichtiger Tierarten an den Klimawandel. Bonn-Bad Godesberg, 277-290. = Naturschutz und Biologische Vielfalt 139.

Doswald, N.; Osti, M. (2011): Ecosystem-based approaches to adaptation and mitigation - good practice examples and lessons learned in Europe. Bonn-Bad Godesberg. = BfN-Skripte 306.

Europäische Kommission (2009): Auswirkungen des Klimawandels auf die Gesundheit von Menschen, Tieren und Pflanzen. Begleitpapier für das Weißbuch - Anpassung an den Klimawandel: Ein europäischer Aktionsrahmen. KOM/2009/147 endgültig. Brüssel.

European Commission (2012): Draft Guidelines on Climate Change and Natura 2000 - Dealing with the impact of climate change on the management of the Natura 2000 Network. Luxemburg.

Fleischhauer, M.; Overbeck, G.; Janssen, G.; Kufeld, W. (2013): Raumplanung und Klimaschutz - ein Überblick. In: Birkmann, J.; Vollmer, M.; Schanze, J. (Hrsg.): Raumentwicklung im Klimawandel. Herausforderungen für die räumliche Planung. Hannover, 90-119. = Forschungsberichte der ARL 2.

Freudenberger, L.; Schluck, M.; Ibisch, P. L. (2012): Bewertung der Funktionstüchtigkeit von Ökosystemen im Klimawandel - neuartige Prioritätensetzung auf der Grundlage aktueller Ökosystemforschung. In: Ibisch, P. L.; Kreft, S.; Luthardt, V. (Hrsg.): Regionale Anpassung des Naturschutzes an den Klimawandel: Strategien und methodische Ansätze zur Erhaltung der Biodiversität und Ökosystemdienstleistungen in Brandenburg. Eberswalde, $144-155$.

Frischbier, N.; Profft, I.; Hagemann, U. (2014): Potential Impacts of Climate Change on Forest Habitats in the Biosphere Reserve Vessertal-Thuringian Forest in Germany. In: Rannow, S.; Neubert, M. (Hrsg.): Managing protected areas in Central and Eastern Europe under climate change. Dordrecht, 243-257. = Advances in Global Change Research 58.

German Commission for UNESCO (2011): For life, for the future. Biosphere reserves and climate change. Bonn.

Gerstengarbe, F.-W.; Werner, P. (1997): Waldbrandentwicklung im Land Brandenburg. In: AFZ/Der Wald 7, 392-394.

Gitay, H.; Finlayson, C. M.; Davidson, N. (2011): A Framework for assessing the vulnerability of wetlands to climate change. Gland. = Ramsar Technical Report 5/CBD Technical Series 57.

Haas, W.; Weisz, U.; Pallua I.; Hutter, H.-P.; Essl, F.; Knoflacher, H.; Formayer, H.; Gerersdorfer, T.; Balas, M. (2010): Handlungsempfehlungen zur Anpassung an den Klimawandel in Österreich. Aktivitätsfelder: Gesundheit, Natürliche Ökosysteme/Biodiversität und Verkehrsinfrastruktur. Wien.

Haase, M. (2014): Gemeine Flußmuschel Unio crassus. In: Kerth, G.; Blüthgen, N.; Dittrich, C.; Dworschak, K.; Fischer, K.; Fleischer, T.; Heidinger, I.; Limberg, J.; Obermaier, E.; Rödel, M.-O.; Nehring, S. (Hrsg.): Anpassungskapazität naturschutzfachlich wichtiger Tierarten an den Klimawandel. Bonn-Bad Godesberg, 45-48. = Naturschutz und Biologische Vielfalt 139 .

Hansen, L. J.; Hoffman, J. R. (2011): Climate Savvy - Adapting Conservation and Resource Management to a Changing World. Washington. 
Hartmann, T.; Spit, T. (2014): Frontiers of land and water governance in urban regions. In: Water International 39, 6, 791-797.

Heller, N. E.; Zavaleta, E. S. (2009): Biodiversity management in the face of climate change: A review of 22 years of recommendations. In: Biological Conservation 142, 1, 14-32.

Hoffmann, A. (2011): Klimawandel und Biodiversität - Folgen für Deutschland, Gewässerökologie. Vortrag am 19./20. Mai 2011 auf der Konferenz "Klimawandel und Biodiversität - Folgen für Deutschland“. Frankfurt am Main.

Ibisch, P. L.; Luthardt, V.; Kreft, S.; Nusko, N.; Strixner, L.; Arndt, P. (2014): Anpassung des Naturschutzes an den Klimawandel in Brandenburg: Empfehlungen für Entscheidungsträger. Eberswalde.

IPCC - Intergovernmental Panel on Climate Change (2007): Climate Change 2007. Impacts, Adaptation and Vulnerability. Working Group II Contribution to the Fourth Assessment Report of the Intergovernmental Panel on Climate Change. Cambridge.

IPCC - Intergovernmental Panel on Climate Change (2012): Managing the risks of extreme events and disasters to advance climate change adaptation. Special Report of the Intergovernmental Panel on Climate Change. Cambridge.

Ivajnšič, D.; Kaligarič, M. (2014): How to preserve coastal wetlands threatened by climate change-driven rises in sea level? In: Environmental Management 54, 4, 671-684.

Jenssen, M.; Hofmann, G.; Pommer, U. (2007): Die natürlichen Vegetationspotentiale Brandenburgs als Grundlage klimaplastischer Zukunftswälder. Beiträge zur Gehölzkunde 2007, 17-29.

Job, H.; Becken, S.; Paeth, H. (2011): Schutzgebiete, Biodiversität und Tourismus - künftige Herausforderungen. In: Natur und Landschaft $86,12,521-526$.

Karczmarzyk, A.; Pfriem, R. (Hrsg.) (2011): Klimaanpassungsstrategien von Unternehmen. Marburg.

Knieling, J.; Müller, B. (Hrsg.) (2015): Klimaanpassung in der Stadtund Regionalentwicklung. Ansätze, Instrumente, Maßnahmen und Beispiele. München.

LAGS - Landesanstalt für Großschutzgebiete (1999): Der Pflege- und Entwicklungsplan (Entwurf) für das Biosphärenreservat Flusslandschaft Elbe - Brandenburg. http://www.lugv.brandenburg.de/ cms/media.php/lbm1.a.3310.de/pep elbe.pdf (17.07.2016).

Ludewig, K.; Hanke, J. M.; Korell, L.; Jensen, K. (2014): Mögliche Auswirkungen des Klimawandels auf die Vegetation von Auenwiesen entlang der mittleren Elbe. In: Prüter, J.; Keienburg, T.; Schreck, C. (Hrsg.): Klimafolgenanpassung im Biosphärenreservat Niedersächsische Elbtalaue - Modellregion für nachhaltige Entwicklung. Hamburg, 27-34. = Berichte aus den KLIMZUGNORD Modellgebieten 5.

Macgregor, N. A.; van Dijk, N. (2014): Adaptation in practice: how managers of nature conservation areas in eastern England are responding to climate change. In: Environmental Management 54, $4,700-719$.

Marzeion, B.; Levermann, A. (2014): Loss of cultural world heritage and currently inhabited places to sea-level rise. In: Environmental Research Letters 9, 3, 1-7.

MLUL - Ministerium für Ländliche Entwicklung, Umwelt und Landwirtschaft des Landes Brandenburg (2015): Managementplan für das FFH-Gebiet 351 „Karthane“ (unveröffentlichter Entwurf). Potsdam.

MLUR - Ministerium für Landwirtschaft, Umweltschutz und Raumordnung des Landes Brandenburg (2002): Biosphärenreservat Flusslandschaft Elbe - Brandenburg. Landschaftsrahmenplan mit integriertem Rahmenkonzept, Band 2: Grundlagen, Bestandsaufnahme, Bewertung. Potsdam.

Morecroft, M. D.; Crick, H. Q. P.; Duffield, S. J.; Macgregor, N. A. (2012): Resilience to climate change: translating principles into practice. In: Journal of Applied Ecology 49, 3, 547-551.

Mosbrugger, V.; Brasseur, G.; Schaller, M.; Stribrny, B. (Hrsg.) (2014): Klimawandel und Biodiversität. Folgen für Deutschland. Darmstadt.
Naumann, S.; Davis, M.; Goeller, B.; Gradmann, A.; Mederake, L.; Stadler, J.; Bockmühl, K. (2015): Ökosystembasierte Ansätze zur Anpassung an den Klimawandel und zum Klimaschutz im deutschsprachigen Raum. Bonn-Bad Godesberg. = BfN-Skripte 395.

Paulini, I.; Haum, R. (2015): Mündige Bürger braucht das Land! Der Beitrag von Bildung und Forschung zur Großen Transformation. In: Politische Ökologie 33, 3, 24-29.

Prüter, J.; Keienburg, T.; Schreck, C. (Hrsg.) (2014): Klimafolgenanpassung im Biosphärenreservat Niedersächsische Elbtalaue - Modellregion für nachhaltige Entwicklung. Hamburg. = Berichte aus den KLIMZUG-NORD Modellgebieten 5.

Rabitsch, W.; Winter, M.; Kühn, E.; Kühn, I.; Essl, F.; Gruttke, H. (2010): Auswirkungen des rezenten Klimawandels auf die Fauna in Deutschland. Bonn-Bad Godesberg. = Naturschutz und biologische Vielfalt 98.

Rannow, S.; Macgregor, N. A.; Albrecht, J.; Crick, H. P. Q.; Förster, M.; Heiland, S.; Janauer, G.; Morecroft, M. D.; Neubert, M.; Sarbu, A.; Sienkiewicz, J. (2014): Managing Protected Areas Under Climate Change: Challenges and Priorities. In: Environmental Management 54, 4, 732-743.

Renn, O. (2014): Das Risikoparadox. Warum wir uns vor dem Falschen fürchten. Frankfurt am Main.

Ritter, E.-H. (2007): Klimawandel - eine Herausforderung an die Raumplanung. In: Raumforschung und Raumordnung 65, 6 , 531-538.

Roers, M.; Wechsung, F. (2015): Neubewertung der Auswirkungen des Klimawandels auf den Wasserhaushalt im Elbegebiet. In: Hydrologie und Wasserbewirtschaftung 59, 3, 109-119.

Scharnke, M.; Krüger, F.; Urban, B.; Schneider, W. (2014): Modellierung von klimainduzierten Veränderungen des Bodenwasserhaushaltes von Aueböden an der unteren Mittelelbe. In: Prüter, J.; Keienburg, T.; Schreck, C. (Hrsg.): Klimafolgenanpassung im Biosphärenreservat Niedersächsische Elbtalaue - Modellregion für nachhaltige Entwicklung. Hamburg. = Berichte aus den KLIMZUG-NORD Modellgebieten 5.

Schliep, R.; Bertzky, M.; Hirschnitz, M.; Stoll-Kleemann, S. (2008) Changing Climate in Protected Areas? Risk Perception of Climate Change by Biosphere Reserve Managers. In: Gaia 17, S1, $116-124$.

Scholz, E. (1962): Die naturräumliche Gliederung Brandenburgs. Potsdam.

Scholz, M.; Henle, K.; Dziock, F.; Stab, S.; Foeckler, F. (Hrsg.) (2009): Entwicklung von Indikationssystemen am Beispiel der Elbaue. Stuttgart.

Scholz, M.; Stab, S.; Dziock, F.; Henle, K. (Hrsg.) (2005): Lebensräume der Elbe und ihrer Auen. Berlin. = Konzepte für die nachhaltige Entwicklung einer Flusslandschaft 4.

Schreck, C.; Katz, C. (2014): KLIMAgespräche - Kommunikation von Klimawissen in Vereinen, Verbänden und Interessengruppen. In: Prüter, J.; Keienburg, T.; Schreck, C. (Hrsg.): Klimafolgenanpassung im Biosphärenreservat Niedersächsische Elbtalaue - Modellregion für nachhaltige Entwicklung. Hamburg, 107-112. = Berichte aus den KLIMZUG-NORD Modellgebieten 5.

Schuster, S. (2014): Kann der Kiebitz Vanellus vanellus den Klimawandel überstehen? Zum Rückgang der Brut- und Mauserbestände im Voralpenraum. In: Vogelwelt 135, 2, 75-82.

Staudt, A.; Leidner, A. K.; Howard, J.; Brauman, K. A.; Dukes, J. S.; Hansen L.J.; Paukert, C.; Sabo, J.; Solórzano, L. A. (2013): The added complications of climate change: understanding and managing biodiversity and ecosystems. In: Frontiers in Ecology and the Environment 11, 9, 494-501.

Stein, B. A.; Staudt, A.; Cross, M. S.; Dubois, N. S.; Enquist, C.; Griffis, R.; Hansen, L. J.; Hellmann, J. J.; Lawler, J. J.; Nelson, E. J.; Pairis, A. (2013): Preparing for and managing change: climate adaptation for biodiversity and ecosystems. In: Frontiers in Ecology and the Environment 11, 9, 502-510. 
Stiens, G. (1977): Zur Verwendung von Szenarien in der Raumplanung. In: Raumforschung und Raumordnung 35, 1/2, 69-73.

UNESCO - United Nations Educational, Scientific and Cultural Organization (1996): Biosphere Reserves: The Seville Strategy and the Statuary Framework of the World Network. Paris.

UNESCO - United Nations Educational, Scientific and Cultural Organization (2008): Madrider Aktionsplan vom 3. April 2008. Auf dem Weltkongress zum MAB-Programm vom 5 . bis 8 . Februar 2008 in Madrid verabschiedet. http://www.unesco.de/ infothek/dokumente/konferenzbeschluesse/madrid-aktionsplan. html (06.07.2016).

UNESCO - United Nations Educational, Scientific and Cultural Organization (2011): Dresdner Erklärung zu Biosphärenreservaten und Klimawandel. http://www.unesco.de/fileadmin/ medien/Dokumente/Wissenschaft/MAB_FINAL_DRESDNER_ ERKLAERUNG.pdf (30.06.2016).

von Haaren, C.; Galler, C. (Hrsg.) (2011): Zukunftsfähiger Umgang mit Wasser im Raum. Hannover. $=$ Forschungs- und Sitzungsberichte der ARL 234.

von Haaren, C.; Palmas, C.; Siewert, A.; Boll, T. (2014): Landschaft: Gesellschaftliche und wissenschaftliche Herausforderungen der Energiewende. In: WSL - Eidgenössische Forschungsanstalt für Wald, Schnee und Landschaft (Hrsg.): Landschaft und Energie- wende. Der Einfluss erneuerbarer Energien auf die Landschaft. Birmensdorf, 25-28. = WSL-Berichte 21.

Wechsung, F.; Hartje, V.; Kaden, S.; Venohr, M.; Hansjürgens, B.; Gräfe, P. (Hrsg.) (2013): Die Elbe im Globalen Wandel: Eine integrative Betrachtung. Berlin. = Konzepte für die nachhaltige Entwicklung einer Flusslandschaft.

Wilke, C.; Rannow, S. (2013): Management Handbook - A Guideline to Adapt Protected Area Management to Climate Change. HABIT-CHANGE Report 5.3.2. http://www.central2013.eu/ fileadmin/user_upload/Downloads/outputlib/HABIT-CHANGE_ 5_3_2_Management_Handbook.pdf (17.07.2016).

Wilke, C.; Rannow, S. (2014): A Methodical Framework for Climate Change-Adapted Management in Protected Areas. In: Rannow, S.; Neubert, M. (Hrsg.): Managing Protected Areas in Central and Eastern Europe Under Climate Change. Dordrecht, 159-172. = Advances in Global Change Research 58.

Zarzo Fuertes, O.; Heiland, S.; Rannow, S.; Wilke, C. (2011): HABITCHANGE. Handbook for Stakeholder Involvement in the CAMP Process. http://habit-change.eu/fileadmin/Dateisammlung/files/ Outputs/HABIT-CHANGE_Stakeholder_Involvement_Handbook_ 2011-12-05.pdf (16.05.2016). 